\title{
Outcome of microsurgical clipping of anterior circulation aneurysms during the period of vasospasm: single center experience in Egypt
}

\author{
Mahmoud M. Taha, Ahmed Alawamry *iD and Tarek H. Abdelbary
}

\begin{abstract}
Objectives: Recent guidelines recommend early closure of the ruptured aneurysms. However, this may not be available in all cases especially in developing countries. This may lead to late surgery during the period of vasospasm which may carry a potential surgical risk. The aim of this study is to report our experience of microsurgical clipping during the period of vasospasm for ruptured anterior circulation aneurysms.

Methods: This study included 36 patients with ruptured anterior circulation aneurysms treated with microsurgical clipping "between January 2014 and December 2016". All patients were operated during the period of vasospasm. We retrospectively reviewed our medical files for any operative complications, any evidence for clinical vasospasm and postoperative ischemia, and for the patients outcome.

Results: Majority of patients were in good World Federation of Neurosurgeons (WFNS) Grades. Thirty (83.3\%) were grades I-III. All the procedures completed successfully except in three patients who had an intraoperative rupture. Clinical vasospasm was reported in 8 (22.2\%) of the patients. Favorable outcome was reported in 31 (86.1\%) patients. We had only one (2.8\%) perioperative mortality from aggravated vasospasm.

Conclusions: Microsurgical clipping of the ruptured anterior circulation aneurysms can be done safely during the period of vasospasm without devastating complications. Securing the aneurysm in patients with late referral in developing countries should be done to prevent rebleeding and to allow for the $3 \mathrm{H}$ therapy application safely.
\end{abstract}

Keywords: Subarachnoid hemorrhage, Aneurysm, Timing of surgery, Vasospasm, Outcome

\section{Introduction}

Overall rebleeding rate of ruptured aneurysms has been reported to be about 36\%, dramatically decreasing the chances of favorable outcome [1]. Together with post-subarachnoid hydrocephalus (20\%), vasospasm and delayed ischemic neurological deficit (symptomatic in $30 \%$ ) represent the main post-ictal complications [2, 3]. Rebleeding differs by being a preventable complication that requires early and complete closure of the ruptured aneurysm [4]. Recent guidelines recommend early and ultra-early securing of the aneurysm by means of surgical clipping or endovascular coiling [4-6]. However, this may not be available for all patients especially in developing countries due to late transportation to specialized neurovascular units. Late admission for patients with aneurysmal subarachnoid hemorrhage during the period of vasospasm forced the neurovascular surgeons to secure the aneurysms during that period which historically carries a bad outcome or postpone the patient till the end of vasospasm period which may carry a high risk of rebleeding. This study aims to report the outcome of microsurgical clipping of anterior circulation aneurysms that were transported late to our center after subarachnoid hemorrhage.

\footnotetext{
* Correspondence: Dr_ahmedalawamry85@yahoo.com

Department of neurosurgery, Zagazig University Hospitals, Zagazig 44519, Egypt
} 


\section{Methods}

We retrospectively reviewed the medical files of all patients with aneurysmal subarachnoid hemorrhage admitted to our department between January 2014 and December 2016. All patients with non-saccular aneurysms, posterior circulation aneurysms, patients treated with endovascular coiling, rebleeding before surgical clipping, and the patients treated early $[5,7,8]$ with microsurgical clipping were excluded. The remaining 36 patients with anterior circulation saccular aneurysms treated with microsurgical clipping during the period of vasospasm between days 4 and 21 were collected and analyzed. The clinical grading of the patients at the time of presentation to our hospital was according to the World Federation of Neurological Surgeons grading system [9]. The majority of cases were good grade (I-III) as shown in (Table 1). The amount of blood at the initial brain computed tomography (CT) was according to Fisher Grading Scale [10]. A diagnostic three-dimensional CT angiography or conventional subtraction angiography was performed after admission. The site and size of the aneurysm were reported. All patients underwent surgical clipping after initial stabilization on the first scheduled operative day. The timing of surgery was classified as intermediate (days 4-14) or late (days after 14) from the day of hemorrhage which was considered as day 0 . The cause of late surgery was due to late transportation to our unit from peripheral centers, unavailable expertise, or unavailable intensive care unit bed in rare cases. Surgical technique included the standard pterional approach for all cases with infrequent temporary clipping according to the surgeon preference. Postoperative patients were transferred to the surgical intensive care unit where $3 \mathrm{H}$ therapy and nimodipine orally or via nasogastric tube in unconscious patients were applied for the rest of the 3 weeks after hemorrhage. Prophylactic phenytoin and steroids were routinely administered in the pre- and postoperative periods. A follow-up brain CT was performed on the first postoperative day and on day 3 to evaluate hematoma on the surgical site, hydrocephalus, or any new ischemic insults (Figs. 1, 2, 3, and 4). Clinical vasospasm was reported in case of development of focal neurological signs or deterioration in the level of consciousness with exclusion of other causes of deterioration such as hydrocephalus, seizures, rebleeding, or electrolyte disturbance. Angioplasty and intra-arterial vasodilator drugs were not applied for the patients with clinical vasospasm. The outcome of the patients was assessed at discharge from the hospital after 3 months using the Glasgow Outcome Scale [11] and was analyzed with variable prognostic factors including vasospasm.

\section{Results}

The age of the patients in this study ranged from 29 to 69 years with a median age of 49.7 years. There was a slight female predominance (56\%) in the study
Table 1 Demographic characteristics of the patients

\begin{tabular}{|c|c|}
\hline \multicolumn{2}{|l|}{ Age } \\
\hline Range & 29-69 years \\
\hline Median & 49.2 years \\
\hline \multicolumn{2}{|l|}{ Gender } \\
\hline Female & $20(56 \%)$ \\
\hline Male & $16(44 \%)$ \\
\hline \multicolumn{2}{|l|}{ WFNS Grading Scale } \\
\hline Grade I & $6(16.7 \%)$ \\
\hline Grade II & $17(47.2 \%)$ \\
\hline Grade III & 7 (19.4\%) \\
\hline Grade IV & $6(16.6 \%)$ \\
\hline Grade V & $0(0 \%)$ \\
\hline \multicolumn{2}{|l|}{ Fisher Grading Scale } \\
\hline Grade I & $0(0 \%)$ \\
\hline Grade II & $18(50 \%)$ \\
\hline Grade III & $16(44.4 \%)$ \\
\hline Grade IV & $2(5.6 \%)$ \\
\hline \multicolumn{2}{|l|}{ Risk factors } \\
\hline Hypertension & $24(66.6 \%)$ \\
\hline Cigarette smoking & $12(33.3 \%)$ \\
\hline Diabetes mellitus & $9(25 \%)$ \\
\hline \multicolumn{2}{|l|}{ Site of aneurysm } \\
\hline Acom & $17(47.2 \%)$ \\
\hline MCA & $10(27.8 \%)$ \\
\hline ICA PCOM & $4(11.1 \%)$ \\
\hline ACA & $5(13.9 \%)$ \\
\hline \multicolumn{2}{|l|}{ Size of the aneurysm } \\
\hline Small $(<6 \mathrm{~m})$ & $4(11.1)$ \\
\hline Medium (6-15 m) & $26(72.2 \%)$ \\
\hline Large (16-24 mm) & $6(16.7 \%)$ \\
\hline \multicolumn{2}{|l|}{ Timing of surgery } \\
\hline Intermediate & $24(66.7 \%)$ \\
\hline Late & 12 (33.3\%) \\
\hline
\end{tabular}

population as shown in Table 1. The majority of cases were of good WFNS grading (I-III) in 30 (83.3\%) of the patients, and the remaining six $(16.7 \%)$ of the patients were grade IV. Eighteen (50\%) of the patients had Fisher grade II, $16(44.4 \%)$ were Fisher grade III, and the remaining two patients were grade IV. Preoperative ventricular drainage was not performed in all patients; however, two (5.6\%) patients developed increased hydrocephalus and required a permanent ventriculoperitoneal shunt. Hypertension, cigarette smoking and diabetes mellitus were the most commonly reported risk factor in $66.7 \%, 33.3 \%$, and $25 \%$ respectively. Ruptured anterior communicating (Acom) artery aneurysm was 

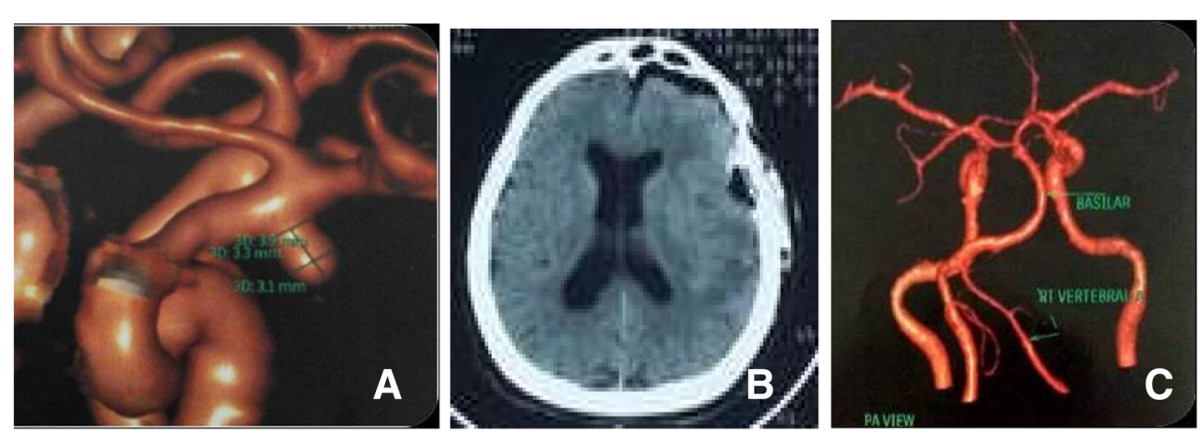

Fig. 1 A 52-year-old male patient with ruptured left PCOM aneurysm. a 3D CTA shows the left PCOM aneurysm. b Follow-up brain CT shows no delayed ischemic insult. c Follow-up 3D CTA shows complete clipping

the commonest site in $17(47.2 \%)$ of the patients followed by ruptured middle cerebral artery (MCA) aneurysm in $10(27.8 \%)$ of the patients. The majority of aneurysms treated were small $(11 \%)$ to medium in size (72\%). Six patients (17\%) had large size aneurysms (16$24 \mathrm{~mm}$ ), and we do not have giant aneurysms in this study. Intermediate surgery was performed in 24 (66.7\%) while the remaining 12 (33.3\%) patients were operated late after day 14 . Clinical vasospasm and delayed ischemic lesions as evident in the follow-up brain CT were reported in eight $(22.2 \%)$ cases. All patients were treated with continued $3 \mathrm{H}$ therapy only as the services of angioplasty or intra-arterial vasodilator injection were not available at our center. Seven patients recovered without devastating neurological deficit, while the remaining case died from aggravated vasospasm. Rebleeding after microsurgical clipping was not reported while postoperative seizures were experienced in two patients. Three-month favorable outcome was reported in 31 (86.1\%) patients; bad outcome in 5 patients included one (2.8\%) patient died from aggravated vasospasm. The most important prognostic factor associated with favorable outcome was the preoperative clinical grading; however, it does not reach statistical significance $(p, 0.421)$. Patients with Fisher grade II had a significant good outcome than other grades $(p, 0.02)$. There was no correlation between preoperative patients' WFNS Grade, Fisher Grade, and the development of vasospasm.

\section{Discussion}

The recent revolution in endovascular intervention with the introduction of detachable coils and flow diversion techniques in the last years leads to the treatment of cerebral aneurysms with endovascular intervention first whenever possible [12, 13]. However; this may not be applicable in many centers in developing countries due to expensive supplies that make surgical clipping the standard treatment modality for ruptured aneurysms.

The optimum timing of microsurgical clipping after aneurysmal subarachnoid hemorrhage was a matter of controversy in the last 5 decades [4]. With the introduction of microsurgical techniques in the 1960s and till 1970s, the majority of neurovascular surgeons practiced late surgery 2 or 3 weeks after hemorrhage. They believed that surgery on swollen and vulnerable brain was dangerous and associated with high rate of complications [7, 14] (Table 2). Whitfield and Kirkpatrick in their Cochrane review in 2001 identified only one randomized
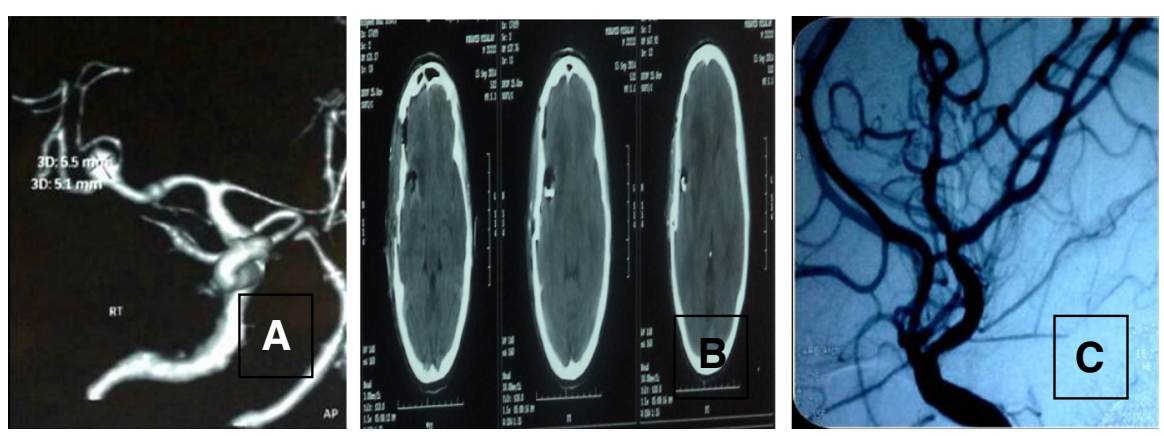

Fig. 2 A 54-year-old female patient with ruptured MCA aneurysm. a 3D CTA shows right MCA aneurysm. b Follow-up brain CT shows no delayed ischemic insult. c Follow-up DSA shows complete clipping 


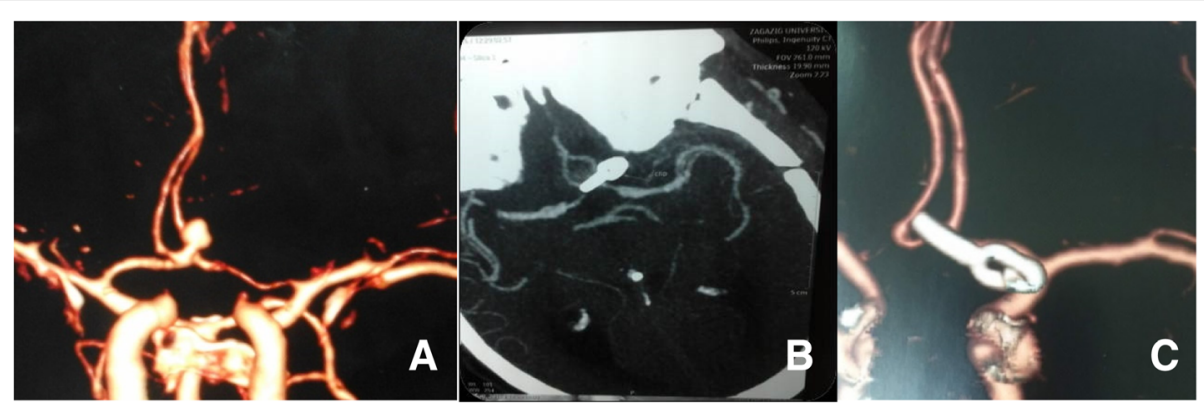

Fig. 3 A 65-year-old male patient with ruptured Acom aneurysm. a 3D CTA shows the ruptured Acom aneurysm. b Follow-up brain CT shows no delayed ischemic insult. c Follow-up 3D CTA shows complete clipping

clinical trial on the timing of surgery after rupture of cerebral aneurysms [6]. In this trial, patients with early surgery (days $0-3$ ) had the best outcome, and patients with intermediate surgery (days 4-7) had the worst outcome [15]. A systemic review that included the aforementioned study and another ten observational studies by de Gans and his colleagues [5] demonstrated that early and intermediate surgical treatment resulted in better outcome than late surgery. Historically, this leads to a popular belief that microsurgical clipping during vasospasm period especially between days 5 and 10 is considered unsafe and associated with high risk of delayed cerebral ischemia $[4,16]$.

However, several recently published studies showed that the outcome was not worse when compared intermediate with the late clipping [4-6]. Dorhout Mees and others evaluated the timing of aneurysm surgery after rupture of cerebral aneurysms in 2016 patients randomized for coiling or clipping within the International Subarachnoid Aneurysmal Trial and supported the current guidelines for early aneurysm treatment in subarachnoid hemorrhage patients. The authors do not recommend postponing treatment in patients between days 5 and 10 after subarachnoid hemorrhage [4]. Orakdogen and others evaluated the timing of surgery with several prognostic factors in patients who underwent microsurgical clipping for ruptured cerebral aneurysms and demonstrated that the rate of mortality was significantly higher in the day 0 period and the rate of mortality did not differ significantly from the other stages of the disease [17]. The aforementioned studies supported the practice of aneurysm treatment once diagnosed even in the period of vasospasm as in our protocol.

On their study about aneurysm surgery, Maurice-Williams and Lafuente found that on existing evidence, the timing of surgery has little if any effect on the overall management results, and soon, this will become a forgotten and irrelevant controversy that was based on the number of patients lost due to ultra-early rebleeding that could not be saved even in early surgery. Adding to that the technical difficulties faced by the surgeon in early surgery [18].

On average of 3 years after subarachnoid hemorrhage, Satzger et al. compared cognitive functions after early and delayed surgery; patients were examined individually with a comprehensive neuropsychological test battery including tests of premorbid intelligence, concept formation, memory, visuomotor speed, aphasia screening, and mood. Early and late patients were well comparable in terms of years of education and level of premorbid intelligence [19].

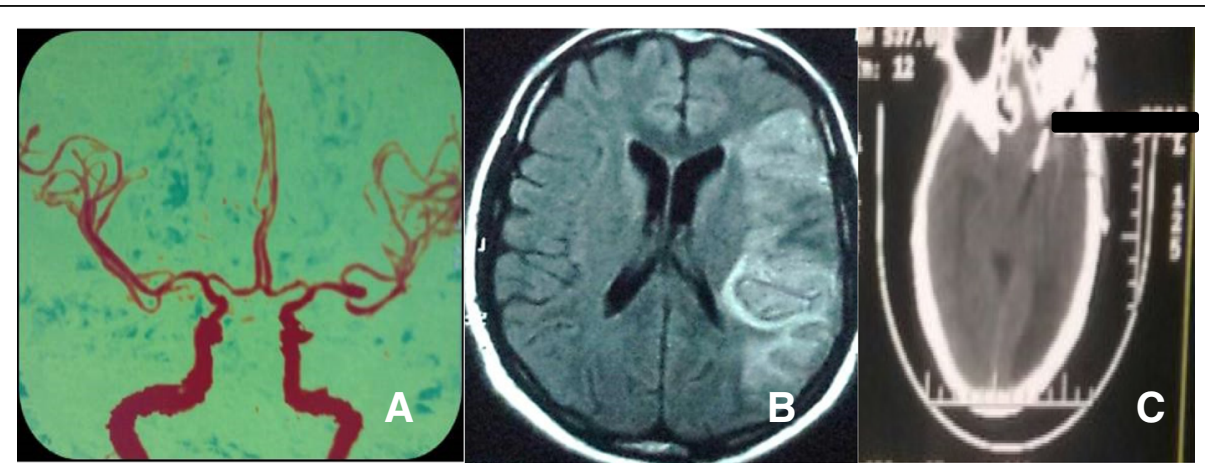

Fig. 4 A 55-year-old female patient with ruptured left MCA aneurysm. a 3D CTA shows the ruptured left MCA aneurysm. b, c Follow-up MRI and brain $C T$ shows aggravated vasospasm 
Table 2 Perioperative complications and outcome

\begin{tabular}{ll}
\hline Perioperative complications & \\
Clinical vasospasm/delayed ischemia & $8(22.2 \%)$ \\
Hydrocephalus & $2(5.6 \%)$ \\
Seizure & $2(5.6 \%)$ \\
Infection & 0 \\
Glasgow Outcome Scale & \\
Favorable outcome (GR + MD) & $31(86.1 \%)$ \\
Bad outcome (SD + PVS) & $4(11.1 \%)$ \\
Death & $1(2.8 \%)$ \\
\hline
\end{tabular}

Symptomatic vasospasm remains a leading cause of delayed morbidity and mortality in patients with aneurysmal subarachnoid hemorrhage [12]. The severity of vasospasm is related to the amount and duration of contact with subarachnoid hemorrhage $[10,20]$. In patients who survive the initial bleeding after rupture of the cerebral aneurysm, about 40 to $70 \%$ developed arterial narrowing with $20-30 \%$ manifested neurological deficit $[1,20]$. Prevention of vasospasm and the delayed cerebral ischemia after ruptured cerebral aneurysms is limited to specific drug treatment and manipulation of blood pressure, intravascular volume, and blood viscosity [3]. Surgical treatment allowed clot removal with irrigation of the basal cisterns which theoretically may reduce the incidence of vasospasm $[1,16]$. It is generally accepted that aneurysm closure with either endovascular coiling or microsurgical clipping facilitates the safe treatment of vasospasm with popular practice of $3 \mathrm{H}$ therapy especially in centers that lack angioplasty [3]. In our study, we observed that preoperative clinical grading was the most important prognostic factor, and patients with good WFNS grade usually had good outcome although it did not reach statistical significance due to the small number of patients. Patients with clinical vasospasm after clipping was associated with poor outcome with one patient died from aggravated vasospasm. The only significant prognostic factor was in patients with Fisher grade II, and all patients had favorable outcome. This is in agreement with other previously published studies investigating the prognostic factors after microsurgical clipping of cerebral aneurysms $[15,17]$. A study by Dilvesi et al. showed that the Fisher Grade is not significant in predicting the intensity of cerebral vasospasm in patients hospitalized with intracranial aneurysm rupture [21]. Orakdogen and others reported that WFNS grade, age, size of the aneurysm, and clinical vasospasm were the most important prognostic factors in patients undergoing surgery after rupture of cerebral aneurysms [17]. Bohnstedt et al. [8] reported that Hunt and Hess grade on admission was the primary prognostic factor of outcome. Samaha et al. [22] demonstrated that preoperative neurological state and the occurrence of arterial vasospasm were the main prognostic factors.

Our study is limited by being retrospective, including small number of patients, evaluating the surgical outcome only and not the overall management for the patients with ruptured anterior circulation aneurysms, and evaluating the patients with delayed cerebral ischemic deficit with brain $\mathrm{CT}$ and not diffusion magnetic resonance image (MRI). However; it presents safe clipping during the period of vasospasm which is a common practice in developing countries due to some logistic difficulties and economic burden. This study demonstrated that patients should not be delayed till the end of vasospasm period and the ruptured aneurysm should be secured whenever applicable to prevent rebleeding and allow treatment of vasospasm with $3 \mathrm{H}$ therapy safely.

\section{Conclusions}

We demonstrated that microsurgical clipping of the ruptured anterior circulation aneurysms can be done safely during the period of vasospasm without devastating complications. Securing the aneurysm in patients with late referral in developing countries should be done to prevent rebleeding and to allow for the $3 \mathrm{H}$ therapy application safely.

\section{Abbreviations \\ Acom: Anterior communicating artery; CT: Computed tomography; MCA: Middle cerebral artery; MRI: Magnetic resonance image; WFNS: World Federation of Neurosurgeons}

\section{Acknowledgements}

Not applicable.

Funding

We do not have personal or institutional financial support for the materials or supplies used in the study.

\section{Availability of data and materials}

Data are available at medical records of department of neurosurgery, Zagazig University, Egypt. The data that support the findings of this study are available on request from the corresponding author [A. Alawamry].

\section{Authors' contributions}

All authors participated in the decision-making regarding the time and type of treatment modality. All authors participated in the microsurgical clipping of aneurysms included in the study. MT and AA participated in the sequence alignment and drafted the manuscript. All authors read and approved the final manuscript.

\section{Ethics approval and consent to participate}

Study design was approved by the ethical committee at faculty of medicine, Zagazig University, Egypt. Date of approval is December 2013. All patients were informed and agreed to participate in this study with written consents.

\section{Consent for publication}

Written consent to publication were obtained from patients whom radiological images were submitted.

Competing interests

The authors declare that they have no competing interests. 


\section{Publisher's Note}

Springer Nature remains neutral with regard to jurisdictional claims in published maps and institutional affiliations.

Received: 10 August 2018 Accepted: 6 January 2019

Published online: 28 January 2019

\section{References}

1. Mahaney KB, Todd MM, Torner JC for the IHAST investigators. Variation of patient characteristics, management, and outcome with timing of surgery for aneurysmal subarachnoid hemorrhage. J Neurosurg. 2011;114:1045-53.

2. Germanwala AV, Huang J, Tamargo RJ. Hydrocephalus after aneurysmal subarachnoid hemorrhage. Neurosurg Clin N Am. 2010;21(2):263-70.

3. Grasso G, Alafaci C, Loch Macdonald R. Management of aneurysmal subarachnoid hemorrhage : state of the art and future prospective. Surg Neurol Int. 2017:8:1-15.

4. Dorhout Mees SM, Molyneux AJ, Kerr RS, Algra A, Rinkel GJ. Timing of aneurysm treatment after subarachnoid hemorrhage. Stroke. 2012;43:2126-9.

5. de Gans K, Nieuwkamp DJ, Rinkel GJ, Algra A. Timing of aneurysm surgery in subarachnoid hemorrhage: a systemic review of the literature. Neurosurgery. 2002;50:336-40.

6. Whitfield PC, Kirkpatrick PJ. Timing of surgery for aneurysmal subarachnoid hemorrhage. Cochrane Database Syst Rev. 2001;2:CD001697.

7. Bohm E, Hugosson R. Experiences of surgical treatment of 400 consecutive ruptured cerebral arterial aneurysms. Acta Neurochir (Wein). 1978;40:33-43.

8. Bohnstedt BN, Nguyen HS, Kulwin CG, Shoja MM, Helbig GM, Leipzig TJ, Payner TD, Cohen-Gadol AA. Outcomes for clip ligation and hematoma evacuation associated with 102 patients with ruptured middle cerebral artery aneurysms. World Neurosurg. 2013;80:335-41.

9. Drake CG, Hunt WE, Sano K, Kassell N, Tesadale G, Pertuiset B, DeVilliers JC. Report of World Federation of Neurological Surgeons Committee on a universal subarachnoid hemorrhage grading scale. J Neurosurg. 1988; 68:985-6.

10. Fisher CM, Kistler JP, Davis JM. Relation of cerebral vasospasm to subarachnoid hemorrhage visualized by computerized tomographic scanning. Neurosurgery. 1980;6:1-9.

11. Jennet $B$, Bond M. Assessment of outcome after severe brain damage. A practical scale. Lancet. 1975;1:480-4.

12. Murayama Y, Malisch T, Guglielmi G, Mawad E, Vinuela F, Duckwiler GR, Gobin YP, Klucznik RP, Martin NA, Frazee J. Incidence of cerebral vasospasm after endovascular treatment of acutely ruptured aneurysm: report of 69 cases. J Neurosurg. 1997:87:830-5.

13. Taha MM, Nakahara I, Higashi T, Iwamuro $Y$, Iwaasa M, Watanabe $Y$, Tsunetoshi K, Munemitsu T. Endovascular embolization vs surgical clipping in treatment of cerebral aneurysms: morbidity and mortality with short-term outcome. Surg Neurol. 2006;66:277-84.

14. Kassel NF, Drake CG. Timing of aneurysm surgery. Neurosurgery. 1982;10: 514-9.

15. Ohman J, Heiskanen O. Timing of operation for ruptured supratentorial aneurysm: a prospective randomized study. J Neurosurg. 1989:70:55-60.

16. Kassel NF, Torner JC, Jane JA, Haley EC Jr, Adams HP. The international cooperative study on the timing of aneurysm surgery. Part 2: surgical results. J Neurosurg. 1990;73:37-47.

17. Orakdogen M, Emon ST, Somay H, Engin T, Ates O, Berkman MZ. Prognostic factors in patients who underwent aneurysmal clipping due to spontaneous subarachnoid hemorrhage. Turk Neurosurg. 2016;26(6):840-8.

18. Maurice-Williams RS, Lafuente J. Intracranial aneurysm surgery and its future. J R Soc Med. 2003;96(11):540-3

19. Satzger W, Niedermeier $N$, Schiinberger $t \mathrm{~J}$, Engel RR, Beck OJ. Timing of operation for ruptured cerebral aneurysm and long-term recovery of cognitive functions. Acta Neurochir (Wiela). 1995:136:168-74.

20. Yao PS, Chen GR, Zheng SF, Kang DZ. Predictors of postoperative cerebral ischemia in patients with ruptured anterior communicating artery aneurysms. World Neurosurg. 2017;103:241-7.

21. Đilvesi $Đ$, Cigić T, Papić V, Horvat I, Karan M, Vuleković P. The Fisher grade in predicting a degree of cerebral vasospasm in patients after intracranial aneurysm rupture. Vojnosanit Pregl. 2016;73(4):349-52.

22. Samaha E, Rizk T, Nohra G, Mohasseb G, Okais N. Intracranial arteria aneurysm: from diagnosis to treatment. A retrospective study of 46 surgically treated cases. J Med Liban. 1998;46:122-5.

\section{Submit your manuscript to a SpringerOpen ${ }^{\circ}$ journal and benefit from:}

- Convenient online submission

- Rigorous peer review

- Open access: articles freely available online

- High visibility within the field

- Retaining the copyright to your article

Submit your next manuscript at $\boldsymbol{\nabla}$ springeropen.com 